\title{
Elena-Brândușa Steiciuc
}

\section{« Et les gens qui ne voulaient pas aller jusqu'au bout ont été envoyés au camp »: Matei Vișnice et la condition de l'écrivain dans les régimes totalitaires}

\begin{abstract}
“AND Those Who Didn't Want to See Things THROUGH Were SENT to the GULAG": MATE VIȘNIEC AND THE CONDITION OF BEING A WRITER IN THE TOTALITARIAN REGIMES

Abstract: Matei Vișniec was exiled in Western Europe before the fall of the totalitarian regime in his native country, Romania. The traumas of his life experience have left an important mark in the writer's conscience as a result of which, after becoming a well-known Francophone writer, he started to denounce this terror in his plays, notably the trilogy that constitutes "the trial of communism through theatre". This article draws attention to Vișniec's ethical attitude, the writing techniques utilized in the trilogy and the way in which intellectuals can deploy resistance in such a terrifying atmosphere
\end{abstract}

Keywords: Totalitarian Regime; Censorship; Matei Vișniec; Communism; Dictatorship; Gulag; The Condition of the Intellectual.

\section{ELENA-BRÂNDUȘA STEICIUC}

Université "Stefan cel Mare », Suceava, Roumanie selenabrandusa@yahoo.com

DOI: 10.24193/cechinox.2020.39.20
F xilé en Occident deux ans avant la Chute de la dictature dans son pays, Matei Vişniec fait une place à part dans sa création - en roumain et en français - à une réflexion sur la condition de l'intellectuel « coincé par l'histoire ». Le totalitarisme communiste, cette terrible expérience politique du $\mathrm{XX}^{\mathrm{e}}$ siècle, en Europe et sur d'autres continents, a commencé par « détruire la substance de l'homme ${ }^{1}$, dans un processus où la terreur s'appuyait sur le lavage des cerveaux, sur l'extermination et l'intimidation des opposants. La « création de l'homme nouveau " avait besoin de robots prêts à exécuter les ordres du parti unique et c'est pourquoi, dans les prisons orwelliennes quétaient devenus les divers pays communistes, la liberté individuelle avait complètement disparu. La propagande, instrument efficace de l'époque, visait surtout à embrigader les écrivains, les artistes, dont le statut pouvait varier, selon leur attitude. On pouvait «signer le pacte» et s'assurer une vie commode, se mettant au service de l'appareil d'endoctrinement et devenant ainsi des mercenaires bien rémunérés de l'art officiel. Mais il y avait aussi 
des cas - bien rares, il est vrai - de résistance devant la tentation, quitte à se voir ensuite voué à la marginalisation, sinon à l'exclusion totale.

Les traumas du système totalitaire laissent un écho profond dans la pensée de Matei Vișniec et, même si les régimes communistes en Europe de l'Est sont tombés l'un après l'autre - comme les pièces d'un jeu de domino -, l'écrivain considère que l'expérience de l'utopie communiste ne doit pas sombrer dans l'oubli au début d'un nouveau millénaire. On le sait, « l'horreur travestie en costume humaniste ", comme il l'appelle, a été le sujet de nombreuses recherches faites avec les instruments des historiens, des sociologues, des anthropologues, et il suffit de se rappeler louvrage collectif Le Livre noir du communisme (1997) qui, malgré les reproches qui lui ont été faits, a le mérite de dresser le bilan sombre des régimes se réclamant du marxisme-léninisme.

Matei Vișniec, quant à lui, dénonce la dictature communiste à sa manière : par la littérature, par la force d'une parole qui, alliée au langage souvent explosif de la scène, fait la radiographie d'un système politique qu'il a eu le malheur de connaitre. La plume du dramaturge fait preuve d'un profond engagement éthique et donne, au tournant des millénaires, trois pièces mémorables écrites premièrement en français et successivement autotraduites en roumain, sur lesquelles va s'appuyer mon analyse : L'Histoire du communisme racontée aux malades mentaux / Istoria comunismului povestită pentru bolnavii mintal (1998) ; Richard III n'aura pas lieu ou scènes de la vie de Meyerhold / Richard al III-lea nu se mai face sau Scene din viața lui Meyerhold (2001) ; De la sensation d'élasticité lorsquion marche sur des cadavres / Despre senzația de elasticitate când păşim peste cadavre (2009).

Dans leur variante roumaine, traduits par l'auteur, ces textes, publiés d'abord indépendamment, au fur et à mesure de leur écriture, sont réunis en une anthologie Procesul comunismului prin teatru - parue chez Humanitas en 2012, dont le titre est significatif quant aux visées profondes de l'auteur. Le préambule qu'il signe, intitulé "N'oublions pas, même si nous pardonnons » en dit long sur son attitude d'intellectuel engagé et lui vaut de la part de Mircea A. Diaconu, pour cette manière d'explorer et de dénoncer un monde invivable, le syntagme « une conscience en action $»^{2}$.

Il serait intéressant de nous pencher sur quelques-unes des idées de ce paratexte, pour mieux comprendre l'investissement personnel de Vişniec et l'enjeu de ces pièces dans l'ensemble de son œuvre où - il ne faut pas l'oublier - la prose est mise, elle aussi, au service de ce procès, surtout par un roman comme Syndrome de panique dans la Ville lumière, paru pendant la même décennie et premièrement en roumain, Sindromul de panică în Oraşul Luminilor (2009). «Je crois qu'il est important de nous rappeler ce que nous avons vécu - et dire ce que nous avons vécu à nos enfants, qui nont pas connu le communisme ", affirme-t-il au début de ce plaidoyer qui établit avec précision la fonction de la mémoire et l'importance du témoignage sur ce «trou noir de l'histoire ». Ayant vécu dans la Roumanie dictatoriale jusqu'à l'âge de 31 ans, il s'assigne le devoir de parler du « délire d'une société totalitaire et schizophrène, avec la touche supplémentaire de grotesque que le couple Ceausescu avait imposée au communisme roumain $»^{3}$.

Matei Vișniec dénonce cette époque invivable à travers des « situations 
dramatiques par lesquelles tout spectateur, qu'il ait vécu ou non le drame du communisme, puisse comprendre d'un point de vue émotionnel, viscéral, l'oppression idéologique et l'absurdité de la notion d'utopie scientifique $»^{4}$. Lurgence de son entreprise lui apparaît d'autant plus visible que la pensée critique de l'Occident se trouve dans une grande impasse et que le communisme est devenu « une histoire oubliée, qui arrive parfois à gêner les philosophes".

Et comment "rafraîchir la mémoire » sinon à travers l'émotion provoquée par la parabole de l'hôpital psychiatrique où l'on se demande : quels sont les fous ? les malades internés, résistant dans l'espace d'une aliénation souvent salvatrice, ou bien les autres, leurs médecins et autres surveillants, soumis à la hiérarchie politique et au dogme du système ? par quel moyen dénoncer "l' accouchement" de l'homme nouveau sinon par la scène cauchemardesque de l'enfant-marionnette qui sort des entrailles de sa mère comme un pantin grotesque, dont le premier geste est d'accuser son père, le metteur en scène Meyerhold, de "se moquer des organes de sécurité de notre pays »? de quelle manière faire comprendre l'atrocité d'un régime qui voulait s'emparer tout d'abord du cerveau et de la plume de lécrivain sinon en montrant celui-ci laminé dans le moulin de la censure et de la rééducation, dans l'espace carcéral, où ses seuls visiteurs sont les apparitions fantasmatiques des écrivains français qu'il avait traduits sans pouvoir les publier?

Lêtre humain sans avenir, sans horizon, enfermé dans cet espace de la domination totale et - surtout - l'artiste, qu'aucune idéologie imposée ne devrait entraver, sont le point focal de cette suite de pièces. Matei Vişniec y filtre lexpérience sombre de plusieurs générations d'intellectuels aux prises avec le pouvoir, dans des formules théâtrales qui font une large place au tragique et au comique, au croisement du grotesque et de l'onirique, au glissement vers le fantastique, dans un style qui porte visiblement l'empreinte du poète.

Les trois personnages principaux des pièces prises en discussion représentent des hypostases du créateur pris dans les rouages de la machine coordonnée en haut lieu et qui brisait, sans droit d'appel, les vies et les consciences. L'Histoire du communisme..., pièce dont les dédicataires sont Daniil Harms et « tous les écrivains morts dans les prisons du pouvoir ", est construite autour de l'expérience de Iouri Petrovski, dans l'Hôpital central des Malades mentaux à Moscou, peu avant la mort du Généralissime.

Ayant un certain prestige dans le monde clos de la dictature soviétique, cet écrivain est invité par le directeur à séjourner parmi les «malades » afin de leur raconter l'histoire du communisme et de la Révolution d'Octobre, tout cela au niveau d'entendement de débiles légers, moyens et profonds, car il y a une véritable " hiérarchie de l'aliénation $»^{5}$ dans cette institution où, selon le directeur, «personne ne doit rester en dehors de la lumière de l'art et de la littérature ». Et le pastiche de Vişniec, dans cette réplique de la scène 2 (tout comme dans beaucoup de scènes des pièces de ce triptyque) reprend intertextuellement un discours qui était courant à l'époque de la terreur, cette langue de bois dont l'hypocrisie n'avait d'égal que le cynisme :

Notre conception scientifique de la société dit que l'homme est au centre de l'attention du parti. Et l'art, la littérature, ont un rôle immense dans la transformation de l'homme, c'est pour 
ça que je me pose la question : et les malades mentaux ? Ne sont-ils pas, eux aussi, des hommes ? Ne doit-on pas les transformer, eux aussi ?6

Pénétrant avec Iouri Petrovski dans cet espace du désespoir, mais - paradoxalement - d'une certaine liberté, le lecteur/ le spectateur apprend, petit à petit, " les effets désastreux de la dictature sur les consciences individuelles, qu'il s'agisse de gens simples, d'opposants politiques ou d'artistes obligés à supporter des privations de liberté de toutes sortes, imposés par le régime ", comme le constate Emilia David ${ }^{7}$.

Jouant le jeu du conformisme, l'écrivain qui sait et voit qu'il est surveillé et manipulé sous diverses formes, instille dans son histoire du communisme des fragments de réalité, qui, comme des épées à double tranchant, ont la fonction de dire ce que tout le monde pense, mais que personne n'ose affirmer. Prenons par exemple la scène 8 , où devant les débiles mentaux légers, surveillés par Katia - l'écrivain évoque « la méthode scientifique » de Felix Djerjinski pour identifier ceux qui ne voulaient pas « aller jusqu'au bout » et les méthodes par lesquelles Staline et ses acolytes pratiquaient l'épuration, avec une conclusion toute simple, quintessence des pratiques totalitaires : «Et ceux qui ne voulaient pas aller jusqu'au bout ont été envoyés au camp ${ }^{8}$.

Iouri Petrovski, reprend lui-même (scène 13), comme un fils fasciné par le grand " père des peuples » des phrases stéréotypes faisant partie du culte de la personnalité à l'époque stalinienne, et il le fait devant les avances de Katia, dont la soumission au parti prend très souvent des formes érotiques :
Le pays avait besoin de vrais livres, sur la manière dont on pouvait produire plus de ciment, plus de charbon, plus d'acier. Et Staline montra aux écrivains comment il fallait écrire des livres vrais sur comment on pouvait produire plus de ciment, plus de charbon, plus d'acier'.

Pourtant, la subversion apparaît petit à petit et devant les fous portant des camisoles de force et encadrés de surveillants costauds, Iouri raconte la collectivisation de l'agriculture, époque où la fondation des kolkhozes avait généré trois types d'attitudes chez les paysans, c'est-à-dire obéissants, mitigés, révoltés, ce qui correspond en fait aux trois catégories de "fous " de l'hôpital, qui - dit la didascalie - « réagissent en mugissant comme un troupeau d'animaux ». Un peu plus loin, dans une scène qui précède la fin, et où le motif de la camisole de force est plus présent que jamais (car c'est le cadeau offert à l'écrivain), la parole appartient plutôt aux «malades" et c'est Koukine, ancien acteur, qui évoque le désastre de la classe paysanne en URSS par une citation attribuée à Staline, métaphore de la terreur envahissante :

Il faudra donc faire les kolkhozes dans leurs cours et c'est dans leurs cours tranchés à vif que nous ferons la transformation socialiste de l'agriculture et c'est dans le cœur même de nos paysans que le pays des soviets va semer le blé dorénavant ${ }^{10}$.

Une autre figure d'artiste enfermé dans la souricière du pouvoir est celle du metteur en scène Meyerhold : peu importe si dans Richard III n'aura pas lieu ou scènes 
de la vie de Meyerhold Matei Vişniec empruntant plutôt la logique du rêve - ne reconstitue pas " de manière linéaire un destin tragique ${ }^{11}$. La trajectoire réelle de cette figure emblématique du théâtre européen n'est pas rigoureusement présentée, car ce n'est pas cela le propos du dramaturge qui cherche, comme dans beaucoup de ses pièces, la fonction symbolique du personnage. Ce qui compte c'est l'atmosphère cauchemardesque dans laquelle l'artiste tente de survivre et la peur omniprésente où le Généralissime et ses émissaires imposent - par leurs directives et par la toute-puissante censure - un art obéissant, « au service du peuple», disent-ils.

Les moyens par lesquels le système « entre dans la tête » de l'artiste sont nombreux et organisés dans un réseau sans faille : il y a des commissions de toutes sortes, aux noms de plus en plus ahurissants (Service public de Déminage Idéologique, La Section de Dépistage des Silences Coupables etc.), pour assurer la surveillance totale de la mise en scène de Richard III, incommode pour le pouvoir, car la pièce shakespearienne jouée dans des costumes modernes pourrait prêter à confusion.

Il y a aussi les proches du metteur-enscène - sa femme, ses parents, ses camarades et même le fils qui vient de naître sous la forme grotesque d'une marionnette ensanglantée -, ce qui n'est pas sans rappeler une pratique réelle des régimes totalitaires. Mais il y a le nec plus ultra, l'autocensure, l'autocritique de l'artiste. Meyerhold, devant la Commission du Ministère de la Culture et de la Propagande, s'accuse luimême d'avoir fait de Richard III un personnage "plutôt sympathique et joyeux ", et qui - dit-il vers la fin de la scène 4 , par un cliché incontournable de la langue de bois de l'époque - avait « échappé à ma vigilance révolutionnaire $»^{12}$.

Le thème du tyran, du pouvoir absolu dans la pièce de Shakespeare que Meyerhold tente sans succès de monter réverbère partout dans la pièce de Vişniec, par un subtil écho intertextuel qui instaure un jeu de miroirs complexe entre le roi anglais (dont les crimes, affirme Meyerhold, scène 16 , « ne sont pas commis au nom d'une grande utopie ») et le Généralissime, symbole absolu d'un mal « emballé dans des milliers de discours sur un monde meilleur $»^{13}$.

En effet, comme toute tyrannie, le totalitarisme - sous sa forme exacerbée dans les pays de l'Est de l'Europe - a non seulement détourné le cours normal de l'histoire, mais il a produit des déviations dangereuses de la nature humaine. Porter le masque de l'adhésion, pratiquer le ketman dont parle Czesław Miłosz dans sa Pensée captive est la norme du comportement dans les pays des "démocraties populaires "; l'essayiste polonais observe que ce qui compte dans ce cas n'est pas ce que la personne dit, mais «ce qu'elle a voulu dire », dans un "style spécial, une certaine terminologie et un rituel linguistique $»^{14}$.

Tout artiste, tout écrivain qui n'entend pas adopter cette stratégie duplicitaire est voué à l'échec sur le plan social et même humain et c'est le cas de Sergiu Penegaru, protagoniste de la pièce qui clôt la trilogie, De la sensation d'élasticité lorsquion marche sur des cadavres. Poète et traducteur de littérature française, il refuse de signer le pacte que lui conseille le Rédacteur-en-chef d'une revue littéraire bucarestoise des années 50, La littérature socialiste.

La censure, représentée par le tout-puissant secrétaire de la commission idéologique 
n'accepte pas ses poèmes surréalistes et provocateurs, ni les auteurs qu'il traduit - Ionesco, Beckett, Gide, Breton - qui sont relégués par le Parti dans un fonds spécial de la bibliothèque de l'École de littérature, réservé à ces formes d'« aberration décadente ", genre « cabinet de monstruosités ». Mis en prison pour un geste qui - dans ce pays du double langage - peut passer pour une immense provocation, Sergiu y rencontre les élites du monde littéraire et artistique roumain (Noica, Steinhardt) qu'il met au courant de l'actualité littéraire et surtout de la plus récente pièce de Ionesco jouée à Paris, $\mathrm{La}$ Cantatrice chauve. La pièce dans la pièce, jouée par les détenus dans leur cellule, est signe des vertus libératrices de l'art. D'ailleurs, le théâtre de Ionesco, découvert par le jeune Matei Vișniec dans la Roumanie communiste " où l'absurde quotidien rivalisait avec le théâtre de l'absurde » était/ est « un outil extrêmement efficace de lutte contre loppression, la bêtise et le dogmatisme idéologique ", comme le dit l'auteur dans l'Avant-propos de cette pièce.

L'art asservi à l'utopie totalitaire, l'écrivain marchant dans les sables mouvants du pouvoir au risque dêtre englouti à tout moment, voilà ce que Vișniec dénonce avec force dans ces trois pièces car, comme il l'affirme dans une interview accordée à Lucretia Birladeanu en 2002 pour la revue Contrafort, cette « réécriture de l'utopie communiste met en lumière le mécanisme rééducateur de toute démarche idéologique ${ }^{15}$.

Soulignons encore une fois la dimension profondément éthique de cette trilogie de Vişniec et de son questionnement sur la condition, la place et le rôle de l'intellectuel dans des régimes totalitaires. Qu'il s'appelle Meyerhold, Noica, Harms ou bien Steinhardt, l'écrivain ou l'artiste est confronté aux mêmes vicissitudes et cette trilogie peut être lue en égale mesure comme une interrogation sur la capacité de l'homme à résister devant le mal et devant la machine qui l'écrase.

"Staline n'est pas mort!", le cri de Iouri Petrovski dans l'hystérie généralisée de l'hospice, est en même temps un signal d'alarme que Matei Vișniec lance à tout horizon : pour rappeler au monde contemporain les victimes d'une idéologie absurde et pour que de telles horreurs ne se répètent pas.

\section{BibLIOGRAPHIE}

Arendt, Hannah, Originile totalitarismului, traduction roumaine par Ion Dur et Mircea Ivănescu, Bucarest, Ed. Humanitas, 1994.

David, Emilia, Consecințele bilingrismului în teatrul lui Matei Vișniec, Bucarest, Ed. Tracus Arte, 2015.

Diaconu, Mircea A., Firul Ariadnei. 10 cărți de proză (și nu numai), Cluj-Napoca, Ed. Eikon, 2014, p. 41-63.

Lungu-Badea, Georgiana, "Osons ôter les masques! Un théâtre, une visée immédiate, une visée suggérée - qu'est-ce qui nous trouble ? ", dans Dialogues francophones, Timișoara, Editura Universității de Vest, 2013, p. 33-45.

Nedelcu-Patureau, Mirela, «Pur și simplu un dramaturg », postface du volume de Matei Visniec, Mansardă la Paris cu vedere spre moarte, Pitești, Paralela 45, 2006, p. 275-282.

Miłosz, Czesłav, Gândirea captivă, traduction roumaine de Constantin Geambașu, Bucarest, Humanitas, 2008. 
Visniec, Matei, Istoria comunismului povestită pentru bolnavii mintal, Bucarest, Aula, 2001.

Visniec, Matei, Richard III n'aura pas lieu ou scènes de la vie de Meyerbold, in Mansardă la Paris cu vedere spre moarte, Pitești, Paralela 45, 2006.

Visniec, Matei, De la sensation d'élasticité lorsquion marche sur des cadavres, Carnières-Morlanwelz, Lansman, 2009.

Visniec, Matei, Sindromul de panică în Orașul Luminilor, Bucarest, Cartea Românească, 2009.

\section{Sitographie}

Interview accordée par Visniec à Lucretia Birladeanu : http://www.contrafort.md/old/2002/97/439.html

\section{NoTES}

1. Hannah Arendt, Originile totalitarismului, traduction roumaine par Ion Dur et Mircea Ivănescu, Bucarest, Ed. Humanitas, 1994, p. 19.

2. Mircea A. Diaconu, Firul Ariadnei. 10 cărți de proză (și nu numai), Cluj-Napoca, Ed. Eikon, 2014, p. 41.

3. Matei Visniec, Procesul comunismului prin teatru, Bucarest, Ed. Humanitas, 2012, p. 13.

4. Ibid., p. 14.

5. Georgiana Lungu-Badea, "Osons ôter les masques ! Un théâtre, une visée immédiate, une visée suggérée - qu'est-ce qui nous trouble ? ", dans Dialogues francophones, Timișoara, Editura Universității de Vest, 2013, p. 39.

6. Matei Visniec, Istoria comunismului povestită pentru bolnavii mintal, Bucarest, Ed. Aula, 2001, p. 35.

7. Emilia David, Consecințele bilingvismului în teatrul lui Matei Vişniec, Bucarest, Ed. Tracus Arte, 2015, p. 22.

8. Matei Visniec, Istoria comunismului, p. 19.

9. Ibid., p. 22.

10. Ibid., p. 34.

11. Mirela Nedelcu-Patureau, "Pur și simplu un dramaturg », postface du volume de M. Visniec, Mansardă la Paris cu vedere spre moarte, Pitești, Paralela 45, 2006, p. 275-282.

12. Matei Visniec, Richard al III-lea nu se mai face sau Scene din viaţa lui Meyerbold, in Mansardă la Paris cu vedere spre moarte, Pitești, Paralela 45, 2006, p. 92.

13. Ibid., p. 130.

14. Czesław Miłosz, Gândirea captivă, traduction roumaine par Constantin Geambașu, Bucarest, Humanitas, 2008, p. 98.

15. Interview accordée par Visniec à Lucretia Birladeanu en 2002 : http://www.contrafort.md/old/2002/97/439. html, page consultée le 30/09/2018. 